\title{
SPECIAL ANNOUNCEMENTS
}

\section{AMERICAN POLITICAL SCIENCE ASSOCATION COMMITTEE APPOINTMENTS}

\section{2}

\section{Executive Committee}

Luther Gulick, Chairman, Institute of Public Administration Pendleton Herring, Social Science Research Council

Edward H. Litchfield, American Political Science Association John D. Millett, Columbia University

Roscoe C. Martin, Syracuse University

Catheryn Seckler-Hudson, The American University

Committee on Nominations

James L. McCamy, Chairman, The University of Wisconsin

James M. Burns, Williams College

Hugh Elsbree, Library of Congress

Cullen B. Gosnell, Emory University

George A. Graham, Princeton University

Joseph P. Harris, University of California (Berkeley)

Program Committee

John D. Millett, Chairman, Columbia University

Committee on Local Arrangements

Julian Park, Chairman, University of Buffalo

John Clarke Adams, University of Buffalo

Clara Brogan, Buffalo

J. Murdoch Dawley, State College for Teachers at Fredonia

Walter A. Kendall, Buffalo

Leonard Lawson, Hobart College

Welles V. Moot, Buffalo

Harold F. Peterson, State College for Teachers at Buffalo

Robert H. Stern, University of Buffalo

Glenn G. Wiltsey, University of Rochester

Committee on Awards

Lloyd M. Short, General Chairman, University of Minnesota

Woodrow Wilson Foundation Panel

Dean E. McHenry, Chairman, University of California (Los Angeles)

Malcolm C. Moos, Johns Hopkins University

Mulford Q. Sibley, University of Minnesota

Franklin D. Roosevelt Foundation Panel

Earl Latham, Chairman, Amherst College

George C. S. Benson, Claremont Men's College

Charles B. Hagen, University of Illinois

Wendell L. Willkie Memorial Panel

Walter H. C. Laves, Chairman, Mutual Security Agency

Clarence A. Berdahl, University of Illinois

Graham H. Stuart, Stanford University

\section{Census Advisory Commitree}

E. Allen Helms, Ohio State University

Arthur N. Holcombe, Harvard University

Claudius $\mathrm{O}$. Johnson, University of Washington 
Earl Latham, Amherst College

Edward H. Litchfield, Cornell University

Joseph McGoldrick, New York City

James K. Pollock, University of Michigan

E. E. Robinson, Stanford University

Richard Scammon, Department of State

George W. Snowden, Bureau of Labor Statistics, State of Louisiana

Committee on Organization

James K. Pollock, Chairman, The University of Michigan

William Anderson, University of Minnesota

Charles M. Kneier, University of Illinois

Harvey C. Mansfield, Ohio State University

Special Committee on Service Voting

Paul T. David, Chairman, Brookings Institution

Robert Cutler, Old Colony Trust Company, Boston

Samuel J. Eldersveld, University of Michigan

Bertram M. Gross, Democratic National Committee

Alexander Heard, University of North Carolina

Edward H. Litchfield, ex officio

Richard Scammon, Department of State

Kathryn H. Stone, League of Women Voters

William B. Prendergast, Secretary, United States Naval Academy

Committee on tee Advancement of Teaching

Claude E. Hawley, Chairman, National Security Resources Board

Phillips Bradley, Syracuse University

Marshall E. Dimock, Bethel, Vermont

Harold M. Dorr, University of Michigan

Andrew E. Nuquist, University of Vermont

John A. Vieg, Pomona College

Ruth G. Weintraub, Hunter College

Committee on American Legislatures

Belle Zeller, Chairman, Brooklyn College

Donald Axelrod, New York State Division of the Budget

Mona Fletcher, Kent State University

W. Brooke Graves, Library of Congress

Frederic H. Guild, Kansas Legislative Council

Joseph P. Harris, University of California (Berkeley)

Lashley G. Harvey, Boston University

Jack F. Isakoff, Illinois Legislative Council

John A. Perkins, University of Delaware

Charles W. Shull, Wayne University

Richard C. Spencer, Coe College

O. Douglas Weeks, University of Texas

Commitree on Citizen Participation in Politics

George H. Williams, Chairman, New York University

Ben A. Arneson, Ohio Wesleyan University

Lawrence H. Chamberlain, Columbia University

Rev. Robert C. Hartnett, A merica Magazine

Estes Kefauver, United States Senate

Dayton D. McKean, Dartmouth College

Josephine M. Pisani, Queens College

Arthur T. Vanderbilt, Supreme Court of New Jersey 
John A. Vieg, Pomona College

Howard White, Miami University (Ohio)

Committee on Eastern Europe and the Soviet Union

Frederick C. Barghoorn, Chairman, Yale University

William B. Ballis, University of Washington

Cyril E. Black, Princeton University

Fritz T. Epstein, Stanford University

Merle Fainsod, Harvard University

Andrew Gyorgy, Yale University

Barrington Moore, Jr., Harvard University

Philip E. Mosely, Columbia University

Louis Nemzer, Ohio State University

N. S. Timasheff, Fordham University

Julian Towster, University of California (Berkeley)

Sergius Yakobson, Library of Congress

Committee on the Far East

Harold S. Quigley, Chairman, University of Minnesota

Hugh Borton, Columbia University

Kenneth W. Colegrove, Northwestern University

William Holland, Institute of Pacific Relations

Paul M. A. Linebarger, School of Advanced International Studies

David N. Rowe, Yale University

Robert A. Scalapino, University of California (Berkeley)

H. Arthur Steiner, University of California (Los Angeles)

George E. Taylor, University of Washington

Amry Vandenbosch, University of Kentucky

Harold M. Vinacke, University of Cincinnati

\section{Committee on International Relations}

Walter H. C. Laves, Chairman, Mutual Security Agency

Thomas C. Blaisdell, University of California (Berkeley)

Frederick S. Dunn, Yale University

John Gange, University of Virginia

Charles E. Martin, University of Washington

Pitman B. Potter, The American University

Walter R. Sharp, Yale University

Shepherd L. Witman, Council on World Affairs

Quincy Wright, University of Chicago

Commitee on Labor-Management Relations

Edward W. Carter, Chairman, University of Pennsylvania

Phillips Bradley, Syracuse University

Lloyd Fisher, University of California (Berkeley)

John J. George, Rutgers University

Lee S. Greene, University of Tennessee

Joseph E. Kallenbach, University of Michigan

Louis C. Kesselman, University of Louisville

Avery Leiserson, University of Chicago

Rollin B. Posey, Northwestern University

Committee on Latin-American Affairs

William S. Stokes, Chairman, University of Wisconsin

George I. Blanksten, Northwestern University

Asher N. Christensen, University of Minnesota 
Charles G. Fenwick, Pan American Union

Russell H. Fitzgibbon, University of California (Los Angeles)

Austin F. Macdonald, University of California (Berkeley)

J. Lloyd Mecham, University of Texas

Arthur P. Whitaker, University of Pennsylvania

Committee on Political Parties

Bertram M. Gross, Chairman, Democratic National Committee

Clarence A. Berdahl, University of Illinois

Hugh A. Bone, University of Washington

Franklin L. Burdette, University of Maryland

Paul T. David, Brookings Institution

Joseph P. Harris, University of California (Berkeley)

Max M. Kampelman, Staff, United States Senate

John W. Lederle, University of Michigan

Norton E. Long, Western Reserve University

E. E. Schattschneider, Wesleyan University

Jasper B. Shannon, University of Kentucky

George H. E. Smith, Staff, United States Senate

\section{Committee on Public Administration}

Wallace S. Sayre, Chairman, College of the City of New York George A. Graham, Princeton University

Charles McKinley, Reed College

Roscoe C. Martin, Syracuse University

Samuel C. May, University of California (Berkeley)

Clarence E. Ridley, International City Managers'Association

Walter R. Sharp, Yale University

Lloyd M. Short, University of Minnesota

Herman M. Somers, Haverford College

Robert A. Walker, Stanford University

Kenneth O. Warner, Civil Service Assembly

Edward W. Weidner, Michigan State College

Committee on Research Methods

Franz L. Neumann, Chairman, Columbia University

William T. R. Fox, Columbia University

Louis B. Hartz, Harvard University

Harold D. Lasswell, Yale University

Don Price, Public Administration Clearing House

Leo Rosten, Rand Corporation

Hans Speier, Rand Corporation

Carl B. Swisher, Johns Hopkins University

David B. Truman, Columbia University

Committee on Science and Technology

Arthur N. Holcombe, Chairman, Harvard University

Robert A. Dahl, Yale University

Harlow J. Heneman, United States Department of State

Eldon L. Johnson, University of Oregon

Albert Lepawsky, University of Alabama

Lyman S. Moore, City Manager, Portland, Maine

Richard Niehoff, University of Tennessee

Wallace S. Sayre, College of the City of New York

E. B. Schulz, Lehigh University

Edward W. Weidner, Michigan State College 
Roland Young, Northwestern University

Committee on Western Europe

William Ebenstein, Chairman, Princeton University

Henry W. Ehrmann, University of Colorado

Mario Einaudi, Cornell University

Herman Finer, University of Chicago

Leslie M. Lipson, University of California (Berkeley)

Dean E. McHenry, University of California (Los Angeles)

Charles E. Micaud, Yale University

Robert G. Neumann, University of California (Los Angeles)

Hans Simons, New School for Social Research

Arnold J. Zurcher, New York University 